Research Paper

\title{
Who benefited most from higher cumulative dose of cisplatin among patients with locally advanced naso- pharyngeal carcinoma treated by intensity-modulated radiation therapy? A retrospective study of 527 cases
}

\author{
Xiaomin $\mathrm{Ou}^{*}$, Tingting $\mathrm{Xu}^{*}$, Xiayun $\mathrm{He}$, Hongmei Ying, Chaosu $\mathrm{Hu}^{\bowtie}$ \\ Department of Radiation Oncology, Fudan University Shanghai Cancer Center, Shanghai, China. Department of Oncology, Shanghai Medical College, Shanghai, \\ China \\ * These two authors contributed to the manuscript equally. \\ $\bowtie$ Corresponding author: Dr. Chaosu Hu, Department of Radiation Oncology, Fudan University Shanghai Cancer Center, 270 Dong'An Road, Shanghai 200032, \\ China Tel: + 8621 64175590; fax: +86 2164174774 Email: hucsu62@163.com \\ (C) Ivyspring International Publisher. This is an open access article distributed under the terms of the Creative Commons Attribution (CC BY-NC) license \\ (https://creativecommons.org/licenses/by-nc/4.0/). See http://ivyspring.com/terms for full terms and conditions.
}

Received: 2017.02.19; Accepted: 2017.06.07; Published: 2017.08.23

\begin{abstract}
Purpose: Our previous study demonstrated the benefit of cumulative dose of cisplatin during the whole treatment on locally advanced nasopharyngeal carcinoma (NPC) treated with various chemotherapy strategies. The purpose of this study is to identify the subgroup of locally advanced NPC who benefits from higher dose of cisplatin, and to clarify whether cumulative dose of cisplatin during the whole treatment brings survival benefit to those treated with concurrent chemoradiotherapy (CCRT).

Materials and methods: This retrospective study enrolled 527 patients with locally advanced NPC treated with intensity-modulated radiation therapy (IMRT) and chemotherapy in our institution from 2009 to 2010 . The median cumulative dose of cisplatin of $300 \mathrm{mg} / \mathrm{m}^{2}$ was chose to be the cutoff value of low and high dose subgroups. Survival curves were estimated using the Kaplan-Meier method. Univariate analysis was conducted using the log-rank test. Multivariate analyses (MVA) were performed using Cox proportional hazards regression model.

Results: With a median follow-up of 54.5 (1-76.7) months, high-dose subgroup had a significant higher distant metastasis-free survival (DMFS) $(82.0 \%$ vs. $76.5 \%, p=0.029)$ and overall survival $(O S)(84.1 \%$ vs. 74.0\%, $\mathrm{p}=0.028$ ). Cumulative dose of cisplatin were demonstrated an independent prognostic factors for DMFS (HR=0.524, 95\% Cl 0.340-0.806) and OS $(H R=0.577,95 \% \mathrm{Cl} 0.373-0.893)$ for the entire cohort upon MVA. As for T1-2N2-3, high-dose subgroup had a trend of better DMFS (85.7\% vs. $76.3 \%$, $P=0.069)$ and a significant improvement in OS (87.8\% vs. $76.3 \%, p=0.041)$. Similarly, in the subgroup of T3-4N2-3, higher dose of cisplatin was associated with higher OS $(80.3 \%$ vs. $52.3 \%, p=0.032)$. Cumulative dose of cisplatin was an independent prognostic factor for DMFS $(\mathrm{HR}=0.483,95 \% \mathrm{Cl}$ 0.292-0.798) and OS (HR=0.429, 95\% Cl 0.258-0.715) for patients with T1-4N2-3 disease upon MVA. However, the benefit of higher dose of cisplatin was not observed in the subgroup of T3-4N0-1. For patients receiving CCRT $(n=278)$, those treated with higher dose of cisplatin had a significantly higher DMFS (87.7\% vs. 75.4\%, $p=0.004)$. The benefit mainly derived from T3-4N2-3 patients treated with CCRT (5y DMFS: $87.9 \%$ vs. $58.2 \%$, $p=0.034$ ). Cumulative dose of cisplatin was associated with a lower risk of distant metastasis $(\mathrm{HR}=0.427,95 \% \mathrm{Cl} 0.228-0.801)$ for patients treated with CCRT upon MVA. Conclusions: Our study identified that patients with N2-3 disease were those benefited from higher cumulative dose. The benefit of higher cumulative dose maintained in those treated with CCRT. The intensity of chemotherapy may be tailored based on various stage subgroups in locally advanced NPC.
\end{abstract}

Key words: Nasopharyngeal carcinoma; chemotherapy; cumulative dose of cisplatin; concurrent chemoradiation; prognosis; intensity-modulated radiation therapy. 


\section{Introduction}

The cumulative dose of cisplatin during chemoradiation has been proven a significant prognostic factor for nasopharyngeal carcinoma (NPC) in the era of conventional two-dimensional radiotherapy (2DRT) [1, 2]. Cumulative dose of cisplatin during concurrent chemoradiotherapy (CCRT) was associated with local control [2] and overall survival [1, 2]. With the application of intensity-modulated radiation therapy (IMRT), local control has been notably improved. The main failure pattern has shifted to be distant metastasis, compared with both local and distant relapse in times of 2DRT [3]. Therefore, the prognostic value of cumulative dose of chemotherapy should be re-evaluated in the setting of IMRT. Currently, the role remains controversial [4-6]. Some reports supported the benefit of higher cumulative dose of cisplatin, mainly in reducing distant metastasis $[4,6]$.

Of note, most of the studies above $[1,2,4,5,7]$ enrolled patients treated with CCRT alone and evaluated the role of total dose of cisplatin during CCRT. However, more and more evidence supported the importance of induction chemotherapy [8, 9]. Hence, it is reasonable to take the cumulative dose of cisplatin during the entire treatment into consideration, not only the part during CCRT. To our knowledge, only one report from our institution has evaluated the prognostic value of cumulative dose of cisplatin during the entire treatment [6]. Our previous study showed that total dose of cisplatin was an independent prognostic factor of disease-free survival, distant metastasis-free survival and overall survival in locally advanced NPC treated with IMRT. However, some later publications [5, 7] challenged that the value of total dose of cisplatin may be inflated by induction or adjuvant chemotherapy in our study. Hence, more evidence is needed to clarify whether total dose of cisplatin during the whole treatment is important for distant control and survival, especially in patients receiving CCRT.

In addition, locally advanced NPC is a heterogeneous entity, for example, the failure patterns of T3-4N0-1 and T1-2N2-3 are very different. It is not sure which subgroup of patients benefited from higher dose of cisplatin. Hence, in this study, we enrolled a cohort of patients with locally advanced NPC, treated with chemotherapy and IMRT in our institution from 2009 to 2010 . The cumulative dose of cisplatin during the entire treatment was calculated and the survival rates between various stage subgroups and dose subgroups were compared. The objective of this study is: a) to identify those who benefits from higher dose of cisplatin; b) to clarify whether cumulative dose of cisplatin during the whole treatment brings survival benefit in locally advanced NPC treated with CCRT.

\section{Materials and Methods}

\section{Patient selection}

Consecutive patients with newly diagnosed, stage III-IVB (6th edition of UICC/AJCC) NPC, treated with definite IMRT and chemotherapy in our institution from January 2009 through 2010, were deemed eligible. Institutional Review Board approval was obtained for retrospective review of patients. Exclusion criteria included: not completed the whole course of radiotherapy, received treatment of molecular targeted therapy, previous malignancy or other concomitant malignant disease.

\section{Clinical staging}

Routine workup comprised complete medical history, physical examination, indirect or fiberoptic endoscopic examination, complete blood counts, liver and renal functions. MRI scans of head and neck were performed to evaluate the extent of the locoregional disease. All the patients were staged using the 6th edition of the AJCC staging system. Chest CT, abdominal sonography or abdominal $\mathrm{CT}$, and bone scintigraphy were performed to exclude distant metastasis. PET/CT was selected by the decision of attending physician, generally in advanced $\mathrm{N}$ stage cases.

\section{Chemotherapy}

Chemotherapy was administrated to the entire cohort of patients, including induction chemotherapy followed by CCRT (46.3\%), induction chemotherapy followed by adjuvant chemotherapy (36.1\%), induction chemotherapy followed by radiation alone $(11.2 \%)$ and CCRT with or without adjuvant chemotherapy $(6.4 \%)$. At that time, our institution was conducting a clinical trial comparing the efficacy and toxicities of induction chemotherapy and CCRT with induction chemotherapy and adjuvant chemotherapy. Thus, these two modalities accounted for most. Induction chemotherapy followed by radiation was administrated to old or medically unfit patients who could not tolerate further chemotherapy.

The regimens of induction and adjuvant chemotherapy included TPF, TP, PF and GP. The TPF protocol consisted of docetaxel $75 \mathrm{mg} / \mathrm{m}^{2} \mathrm{IV}$ on day 1 , cisplatin75 mg/m² IV on day 1 , and $5-\mathrm{fu} 500 \mathrm{mg} / \mathrm{m}^{2} \mathrm{~d}$ continuously IV on day1-5. The TP protocol consisted of docetaxel $75 \mathrm{mg} / \mathrm{m}^{2} \mathrm{IV}$ on day 1 , cisplatin75 $\mathrm{mg} / \mathrm{m}^{2}$ IV on day 1 . The PF protocol comprised cisplatin $75 \mathrm{mg} / \mathrm{m}^{2} \mathrm{IV}$ on day 1 and $5-\mathrm{fu} 500 \mathrm{mg} / \mathrm{m}^{2} \mathrm{~d}$ continuously IV on day $1-5$. The GP regimen included cisplatin $75 \mathrm{mg} / \mathrm{m}^{2} \mathrm{IV}$ on day 1 and gemcitabine 1000 
$\mathrm{mg} / \mathrm{m}^{2} \mathrm{IV}$ on day 1,8 . The regimens were repeated every 3 weeks for 2-3 cycles for induction chemotherapy and every 4 weeks for 2-3 cycles for adjuvant phase. Concurrent chemotherapy consisted of cisplatin $40 \mathrm{mg} / \mathrm{m}^{2}$ IV weekly or cisplatin 80 $\mathrm{mg} / \mathrm{m}^{2}$ every 3 weeks during radiation.

\section{Radiation techniques}

Patients were immobilized in the supine position with a thermoplastic mask. CT was performed after immobilization, obtaining 3-mm slices from the anterior clinoid process to the hyoid bone, and 5-mm slices from the hyoid bone to $2 \mathrm{~cm}$ below the sternoclavicular joint. The target volumes were outlined on each layer of the CT images on an IMRT workstation. The gross tumor volume (GTV) included primary tumor and metastatic lymph nodes. The details of the delineation of high-risk clinical target volume (CTV) of primary tumor and cervical lymph nodes were described in our previous publication [6]. A margin of 3-5 $\mathrm{mm}$ around GTV and CTV should be added to account for the patient motion and set-up error. A simultaneous integrated boost method was used. 66 to 70.4 Gy was prescribed to primary tumor in 30-32 fractions. 66Gy was delivered to the metastatic nodes in 30-32 fractions. The high-risk and low-risk CTV received 60Gy and 54Gy, respectively.

At the end of radiotherapy, MRIs of head and neck were conducted. Based on clinical and radiologic examination, nasopharyngeal and neck nodal residual diseases were determined. Local residual diseases were treated by either small-field IMRT or intracavitary afterloading treatment. Small-field IMRT was applied to treat local residual disease just after the planned treatment with 2.2-4.4Gy in one or two daily fractions. Intracavitary afterloading treatment with iridium-192 was used to address local persistence at 2 to 3 weeks after external radiation with 8 to 16 Gy by one or two weekly fractions. Palpable residual nodes present after external radiaiton were treated with a boost of 4-6 Gy in 2 or 3 daily fractions using an electron field of 9 to $12 \mathrm{MeV}$ just after the planned treatment.

\section{Statistical analysis}

SPSS version 22 was used for analysis. The $\mathrm{Chi}^{2}$ test and Fisher's exact test were used to compare baseline characteristics between low-dose (cumulative dose $<300 \mathrm{mg} / \mathrm{m}^{2}$ ) and high-dose (cumulative dose $\geq 300 \mathrm{mg} / \mathrm{m}^{2}$ ) subgroups. The $\mathrm{Chi}^{2}$ test was used to analyze the failure patterns between different stage subgroups (T3-4N0-1M0, T1-2N2-3M0, T3-4N2-3M0). Survival curves were estimated using the Kaplan-Meier method. Overall survival (OS), distant metastasis-free survival (DMFS) and local recurrence-free survival (LRFS) and regional recurrence-free survival (RRFS) were calculated. All the endpoints were defined as the interval from the date of initiation of radiation to the date of the failure or last follow-up. Univariate analysis was conducted using the log-rank test. Given the confounding effect of stage subgroups on survival, we evaluated the survival rates between low-dose and high-dose subgroup using log-rank test classified by stage subgroups (T3-4N0-1M0, T1-2N2-3M0, T3-4N2-3M0). Multivariate analyses were conducted using Cox proportional hazards regression model. The potential prognostic factors were considered in the model, including: age $(\leq 48 />48)$, gender (male/female), stage subgroup (classified parameter: T3-4N0-1/T1-2N2-3/ T3-4N2-3), treatment strategy (induction chemotherapy + radiation/CCRT with or without adjuvant chemotherapy/induction chemotherapy + CCRT/ induction + adjuvant chemotherapy), cumulative dose of DDP $\left(<300 \mathrm{mg} / \mathrm{m}^{2} / \geq 300 \mathrm{mg} / \mathrm{m}^{2}\right)$ and radiation boost (no/yes). Statistical significance was defined as $p<0.05$ based on two-sided tests.

Table 1 Patient characteristic $(\mathrm{N}=527)$

\begin{tabular}{lll}
\hline Factor & Number & Percentage (\%) \\
\hline Age (Median, range) & $48(7-76)$ & \\
Gender Male & 389 & 73.8 \\
$\quad$ Female & 138 & 26.2 \\
T T1 & 83 & 15.7 \\
T2 & 123 & 23.3 \\
T3 & 209 & 39.7 \\
T4 & 112 & 21.3 \\
N N0 & 33 & 6.3 \\
N1 & 146 & 27.7 \\
$\quad$ N2 & 240 & 45.5 \\
$\quad$ N3 & 108 & 20.5 \\
Stage III & 313 & 59.4 \\
IVA & 106 & 20.1 \\
IVB & 108 & 20.5 \\
Pathology II/III & 527 & 100 \\
IMRT & 527 & 100 \\
RT Boost Primary & 24 & 4.6 \\
$\quad$ Nodal & 54 & 10.2 \\
RT Break >3day & 43 & 8.2 \\
Treatment strategy & & \\
Induction CT + RT & 59 & 11.2 \\
Induction CT + CCRT & 244 & 46.3 \\
Induction CT + Adjuvant CT & 190 & 36.1 \\
CCRT + - Adjuvant CT & 34 & \\
\hline Abbreviation: RT=radiation; CT=chemotherapy; CCRT=concurrent chemoradiation.
\end{tabular}

\section{Results}

\section{Baseline characteristics}

A total of 527 patients with locally advanced NPC were enrolled in this study. The baseline characteristics were listed in Table 1. Since the median cumulative cisplatin dose during the entire treatment was $300 \mathrm{mg} / \mathrm{m}^{2}$, we use $300 \mathrm{mg} / \mathrm{m}^{2}$ as the cutoff value to divide patients into low and high dose subgroups. 
The patients of different age and stage were equally distributed in the low-dose and high-dose subgroups, despite a higher proportion of male patients and a marginal higher proportion of radiation boost in the high-dose subgroup (Table 2).

Table 2 Baseline characteristics by difference dose subgroups during the entire treatment

\begin{tabular}{|c|c|c|c|c|c|}
\hline \multirow[t]{2}{*}{ Factor } & Number & $\begin{array}{l}\text { Percentage } \\
(\%)\end{array}$ & Number & $\begin{array}{l}\text { Percentage } \\
(\%)\end{array}$ & $\begin{array}{l}\mathrm{P} \\
\text { value }\end{array}$ \\
\hline & \multicolumn{2}{|c|}{$\begin{array}{l}\text { Total dose of } \\
\mathrm{DDP}<300 \mathrm{mg} / \mathrm{m}^{2}\end{array}$} & \multicolumn{3}{|c|}{ Total dose of DDP $\geq 300 \mathrm{mg} / \mathrm{m}^{2}$} \\
\hline Age $<48 y$ & 89 & 46.8 & 176 & 52.2 & 0.235 \\
\hline$\geq 48 y$ & 101 & 53.2 & 161 & 47.8 & \\
\hline Gender Male & 125 & 65.8 & 264 & 78.3 & $0.002^{*}$ \\
\hline Female & 65 & 34.2 & 73 & 21.7 & \\
\hline $\mathrm{T} 1$ & 27 & 14.2 & 56 & 16.6 & 0.893 \\
\hline $\mathrm{T} 2$ & 45 & 23.7 & 78 & 23.1 & \\
\hline $\mathrm{T} 3$ & 78 & 41.1 & 131 & 38.9 & \\
\hline $\mathrm{T} 4$ & 40 & 21.1 & 72 & 21.4 & \\
\hline $\mathrm{N} \quad \mathrm{N} 0$ & 15 & 7.9 & 18 & 5.3 & 0.119 \\
\hline N1 & 62 & 32.6 & 84 & 24.9 & \\
\hline N2 & 77 & 40.5 & 163 & 48.4 & \\
\hline N3 & 36 & 18.9 & 72 & 21.4 & \\
\hline Stage III & 117 & 61.6 & 196 & 58.2 & 0.724 \\
\hline IVA & 37 & 19.5 & 69 & 20.5 & \\
\hline IVB & 36 & 18.9 & 72 & 21.4 & \\
\hline Pathology II/III & 190 & 100 & 337 & 100 & N.A. \\
\hline RT Boost Yes & 18 & 9.5 & 52 & 15.4 & 0.053 \\
\hline No & 172 & 90.5 & 285 & 84.6 & \\
\hline
\end{tabular}

\section{Survival and Failure Patterns}

With a median follow-up of 54.5(1-76.7) months, 54 patients experienced local recurrence, 32 regional failures, 89 distant metastasis. At the time of analysis, 85 patients were dead. The estimated 5-year LRFS, RRFS, DMFS, DFS were $86.8 \%, 92.2 \%, 80.0 \%$ and $68.4 \%$, respectively. The 5-year estimated OS was $80.7 \%$

Given that locally advanced NPC is a heterogeneous entity, we analyzed the failure patterns by different TN subgroups: T3-4N0-1, T1-2N2-3 and T3-4N2-3 (Table 3). Of patients with T3-4N0-1, the percentage of local relapse and distant metastasis was similar $(11.7 \%$ vs. $12.8 \%)$. As for $\mathrm{T} 3-4 \mathrm{~N} 2-3$, the percentage of distant metastasis was three times of local recurrence $(23.2 \%$ vs. $7.7 \%)$.

\section{The influence of cumulative dose of cisplatin for the entire cohort}

The influence of cumulative dose of cisplatin on LRFS, RRFS, DMFS and OS were summarized in Table 4. For the whole cohort of patients, those treated with higher dose of cisplatin had a significant higher DMFS $(82.0 \%$ vs. $76.5 \%, \mathrm{p}=0.029$, figure $1 \mathrm{~A})$ and OS $(84.1 \%$ vs. $74.0 \%, \mathrm{p}=0.028$, figure $2 \mathrm{~A})$. There was no significant difference in terms of local control and regional control.

Table 3 Failure patterns by different stage subgroups of locally advanced nasopharyngeal carcinoma

\begin{tabular}{|c|c|c|c|c|c|c|c|}
\hline \multirow[t]{2}{*}{ Failure patterns } & \multicolumn{2}{|l|}{ T3-4N0-1 } & \multicolumn{2}{|l|}{ T1-2N2-3 } & \multicolumn{3}{|l|}{ T3-4N2-3 } \\
\hline & Number & Percentage & Number & Percentage & Number & Percentage & P value \\
\hline Local relapse & 21 & $11.7 \%$ & 22 & $10.7 \%$ & 11 & $7.7 \%$ & 0.488 \\
\hline Regional relapse & 5 & $2.8 \%$ & 19 & $9.2 \%$ & 8 & $5.6 \%$ & $0.030^{*}$ \\
\hline Distant metastasis & 23 & $12.8 \%$ & 33 & $16.0 \%$ & 33 & $23.2 \%$ & $0.043^{*}$ \\
\hline Death & 24 & $13.4 \%$ & 31 & $15.0 \%$ & 30 & $21.1 \%$ & 0.151 \\
\hline
\end{tabular}

* indicated $\mathrm{p}<0.05$. The incidence of regional relapse and distant metastasis differed significantly between different stage subgroups.

Table 4 The impact of cumulative dose of cisplatin on the clinical outcomes of the entire cohort $(\mathrm{N}=527)$ and those treated with concurrent chemoradiation $(\mathrm{N}=278)$

\begin{tabular}{|c|c|c|c|c|c|c|c|c|}
\hline Survival/group & $\mathrm{All}^{\sharp}$ & P value & $\mathrm{T} 1-2 \mathrm{~N} 2-3$ & P value & T3-4N0-1 & P value & T3-4N2-3 & $P$ value \\
\hline \multicolumn{9}{|l|}{ Entire cohort } \\
\hline \multicolumn{9}{|l|}{ LRFS } \\
\hline$<300 \mathrm{mg} / \mathrm{m}^{2}$ & $88.0 \%$ & 0.554 & $91.6 \%$ & 0.455 & $85.1 \%$ & 0.697 & $88.0 \%$ & 0.732 \\
\hline$\geq 300 \mathrm{mg} / \mathrm{m}^{2}$ & $86.3 \%$ & & $85.9 \%$ & & $84.1 \%$ & & $89.6 \%$ & \\
\hline \multicolumn{9}{|l|}{ RRFS } \\
\hline$<300 \mathrm{mg} / \mathrm{m}^{2}$ & $90.2 \%$ & 0.336 & $83.3 \%$ & 0.192 & $98.3 \%$ & 0.324 & $87.3 \%$ & 0.441 \\
\hline$\geq 300 \mathrm{mg} / \mathrm{m}^{2}$ & $93.2 \%$ & & $91.4 \%$ & & $94.9 \%$ & & $94.0 \%$ & \\
\hline \multicolumn{9}{|l|}{ DMFS } \\
\hline$<300 \mathrm{mg} / \mathrm{m}^{2}$ & $76.5 \%$ & $0.029^{* *}$ & $76.3 \%$ & $0.069^{*}$ & $82.7 \%$ & 0.502 & $66.0 \%$ & 0.226 \\
\hline$\geq 300 \mathrm{mg} / \mathrm{m}^{2}$ & $82.0 \%$ & & $85.7 \%$ & & $83.9 \%$ & & $74.9 \%$ & \\
\hline \multicolumn{9}{|l|}{ OS } \\
\hline$<300 \mathrm{mg} / \mathrm{m}^{2}$ & $74.0 \%$ & $0.028^{* *}$ & $76.3 \%$ & $0.041^{* *}$ & $85.4 \%$ & 0.654 & $52.3 \%$ & $0.032^{* *}$ \\
\hline$\geq 300 \mathrm{mg} / \mathrm{m}^{2}$ & $84.1 \%$ & & $87.8 \%$ & & $83.1 \%$ & & $80.3 \%$ & \\
\hline \multicolumn{9}{|c|}{ Concurrent chemoradiation cohort } \\
\hline \multicolumn{9}{|l|}{ LRFS } \\
\hline$<300 \mathrm{mg} / \mathrm{m}^{2}$ & $88.4 \%$ & 0.407 & $90.5 \%$ & 0.717 & $87.3 \%$ & 0.315 & $88.9 \%$ & 0.719 \\
\hline$\geq 300 \mathrm{mg} / \mathrm{m}^{2}$ & $86.9 \%$ & & $87.9 \%$ & & $80.1 \%$ & & $93.3 \%$ & \\
\hline RRFS & & & & & & & & \\
\hline
\end{tabular}




\begin{tabular}{|c|c|c|c|c|c|c|c|c|}
\hline$<300 \mathrm{mg} / \mathrm{m}^{2}$ & $88.2 \%$ & 0.172 & $73.9 \%$ & $0.046^{* *}$ & $97.0 \%$ & 0.731 & $100 \%$ & 0.534 \\
\hline$\geq 300 \mathrm{mg} / \mathrm{m}^{2}$ & $93.8 \%$ & & $91.6 \%$ & & $94.7 \%$ & & $96.0 \%$ & \\
\hline \multicolumn{9}{|l|}{ DMFS } \\
\hline$<300 \mathrm{mg} / \mathrm{m}^{2}$ & $75.4 \%$ & $0.004^{* *}$ & $76.8 \%$ & 0.177 & $78.4 \%$ & 0.075 & $58.2 \%$ & $0.034^{* *}$ \\
\hline$\geq 300 \mathrm{mg} / \mathrm{m}^{2}$ & $87.7 \%$ & & $84.7 \%$ & & $91.9 \%$ & & $87.9 \%$ & \\
\hline \multicolumn{9}{|l|}{ OS } \\
\hline$<300 \mathrm{mg} / \mathrm{m}^{2}$ & $76.9 \%$ & 0.513 & $80.7 \%$ & 0.615 & $80.4 \%$ & 0.783 & $60.0 \%$ & 0.182 \\
\hline$\geq 300 \mathrm{mg} / \mathrm{m}^{2}$ & $85.4 \%$ & & $86.5 \%$ & & $82.2 \%$ & & $87.3 \%$ & \\
\hline
\end{tabular}

\# The comparison of survival rate was performed by Log-rank test (classified by various stage-subgroups).

${ }^{* *}$ indicated $\mathrm{p}<0.05 .{ }^{*}$ indicated marginal significance.

Table 5 Multivariate analyses of various clinical factors on survival rates of the entire cohort and the T1-4N2-3 subgroup

\begin{tabular}{|c|c|c|c|c|c|c|c|}
\hline Survival/factor & $\mathrm{P}$ & HR & $95 \% \mathrm{CI}$ & Survival/factor & $\mathrm{P}$ & HR & $95 \% \mathrm{CI}$ \\
\hline Entire cohort & & & & T1-4N2-3 & & & \\
\hline LRFS & & & & LRFS & & & \\
\hline Gender & 0.041 & 0.451 & $0.210-0.968$ & Age & 0.007 & 0.317 & $0.137-0.734$ \\
\hline \multirow[t]{2}{*}{ RT Boost } & 0.004 & 2.400 & $1.317-4.376$ & Gender & 0.037 & 0.317 & $0.108-0.934$ \\
\hline & & & & RT Boost & 0.001 & 3.516 & $1.704-7.257$ \\
\hline RRFS & & & & RRFS & & & \\
\hline RT Boost & 0.002 & 3.217 & $1.540-6.723$ & RT Boost & 0.000 & 4.291 & $1.984-9.280$ \\
\hline DMFS & & & & DMFS & & & \\
\hline Gender & 0.002 & 0.384 & $0.207-0.710$ & Gender & 0.010 & 0.406 & $0.205-0.804$ \\
\hline Stage subgroup & 0.014 & & & Stage subgroup & 0.022 & 0.564 & $0.345-0.921$ \\
\hline T3-4N2-3M0 & & 1.0 & N.A. & T3-4N2-3M0 & & 1.0 & N.A. \\
\hline T3-4N0-1M0 & 0.007 & 0.477 & $0.278-0.817$ & T1-2N2-3M0 & 0.022 & 0.564 & $0.345-0.921$ \\
\hline T1-2N2-3M0 & 0.026 & 0.574 & $0.352-0.935$ & & & & \\
\hline Total DDP dose & 0.003 & 0.524 & $0.340-0.806$ & Total DDP dose & 0.004 & 0.483 & $0.292-0.798$ \\
\hline RT Boost & 0.019 & 1.860 & $1.108-3.123$ & RT Boost & 0.021 & 1.962 & $1.105-3.484$ \\
\hline OS & & & & OS & & & \\
\hline Age & 0.025 & 1.662 & $1.068-2.558$ & Stage subgroup & 0.036 & 0.580 & $0.348-0.965$ \\
\hline Stage subgroup & 0.033 & & & T3-4N2-3M0 & & 1.0 & N.A. \\
\hline T3-4N2-3M0 & & 1.0 & N.A. & T1-2N2-3M0 & 0.036 & 0.580 & $0.348-0.965$ \\
\hline T3-4N0-1M0 & 0.015 & 0.506 & $0.292-0.877$ & Total DDP dose & 0.001 & 0.429 & $0.258-0.715$ \\
\hline T1-2N2-3M0 & 0.042 & 0.591 & $0.356-0.982$ & & & & \\
\hline Total DDP dose & 0.014 & 0.577 & $0.373-0.893$ & & & & \\
\hline
\end{tabular}

Age $(>48 / \leq 48)$, gender $(\mathrm{F} / \mathrm{M})$, Stage subgroup (classified parameter), treatment strategy (induction chemotherapy radiation/induction chemotherapy + CCRT/induction and adjuvant chemotherapy/ CCRT + - adjuvant chemotherapy), Cumulative dose of DDP $\left(\geq 300 \mathrm{mg} / \mathrm{m}^{2} /<300 \mathrm{mg} / \mathrm{m}^{2}\right)$, radiation boost (yes/no) were included into multivariate analysis.

Abbreviation: $\mathrm{RT}$. $=$ Radiation; $\mathrm{DDP}=$ cisplatin.

Table 5 summarized multivariate analyses. After controlling of confounding factors, gender, stage subgroup, cumulative dose of cisplatin and radiation boost were significant for DMFS. Age, stage subgroup and cumulative dose of cisplatin were independent prognostic factors for OS. Cumulative dose of cisplatin was associated with lower risk of distant metastasis (HR $=0.524,95 \%$ CI $0.340-0.806)$ and death (HR=0.577, 95\% CI 0.373-0.893).

\section{The influence of cumulative dose of cisplatin for various stage subgroups}

The impact of cumulative dose of cisplatin was further analyzed by various stage subgroups (Table 4). As for T1-2N2-3, those treated with higher dose of cisplatin had a trend of better DMFS $(85.7 \%$ vs. $76.3 \%$, $\mathrm{p}=0.069$, figure 1C) and a significant improvement in OS ( $87.8 \%$ vs. $76.3 \%, \mathrm{p}=0.041$, figure 2 C). Similarly, in the subgroup of T3-4N2-3, higher dose of cisplatin was associated with higher OS $(80.3 \%$ vs. $52.3 \%$, $\mathrm{p}=0.032$, figure 2D). However, the benefit of higher dose of cisplatin was not observed in the subgroup of T3-4N0-1 (figure 1B and figure 2B).
Multivariate analyses were further performed in the subgroups of T1-4N2-3 (Table 5) and T3-4N0-1. As for T1-4N2-3, gender, stage group, cumulative dose of cisplatin and radiation boost were significant for DMFS upon multivariate analysis. Cumulative dose and stage subgroup were independent prognostic factors for OS. Cumulative dose of cisplatin was associated with lower risk of distant metastasis $(\mathrm{HR}=0.483,95 \% \mathrm{CI} 0.292-0.798)$ and death $(\mathrm{HR}=0.429$, $95 \% \mathrm{CI} 0.258-0.715)$ for patients with T1-4N2-3 disease. As for T3-4N0-1, we could not identify any independent prognostic factor for local control, regional control, distant metastasis and overall survival.

\section{The influence of cumulative dose of cisplatin for those treated with CCRT}

A total of 278 patients received CCRT. Among them, 244 received induction chemotherapy followed by CCRT, 34 were treated with CCRT with/without adjuvant chemotherapy. The median cumulative dose of cisplatin was $310 \mathrm{mg} / \mathrm{m}^{2}$. In order to keep consistency with previous analysis, we used 
$300 \mathrm{mg} / \mathrm{m}^{2}$ as the cutoff value to divide the patients into low-dose and high-dose subgroup.

For the whole cohort of patients $(n=278)$, those treated with higher dose of cisplatin had a significantly higher DMFS $(87.7 \%$ vs. $75.4 \%, \mathrm{p}=0.004$, figure 3A, Table 4). The benefit of higher dose of cisplatin was most prominent in the subgroup of T3-4N2-3. In this subgroup, higher dose of cisplatin was notably associated with higher DMFS $(87.9 \%$ vs. $58.2 \%, \mathrm{p}=0.034$, figure $3 \mathrm{~B}$ ). In multivariate analysis incorporating various clinical parameters (Table 6), cumulative dose of cisplatin was demonstrated to be an independent prognostic factor for DMFS in those treated with CCRT $(p=0.008, H R=0.427,95 \% \mathrm{CI}$ : $0.228-0.801)$. Furthermore, in the multivariate analysis among those treated with induction chemotherapy plus CCRT, higher cumulative dose of cisplatin was also a favorable prognostic factor for DMFS $(\mathrm{p}=0.005$, HR=0.372, 95\%CI: 0.186-0.743, Table 6).
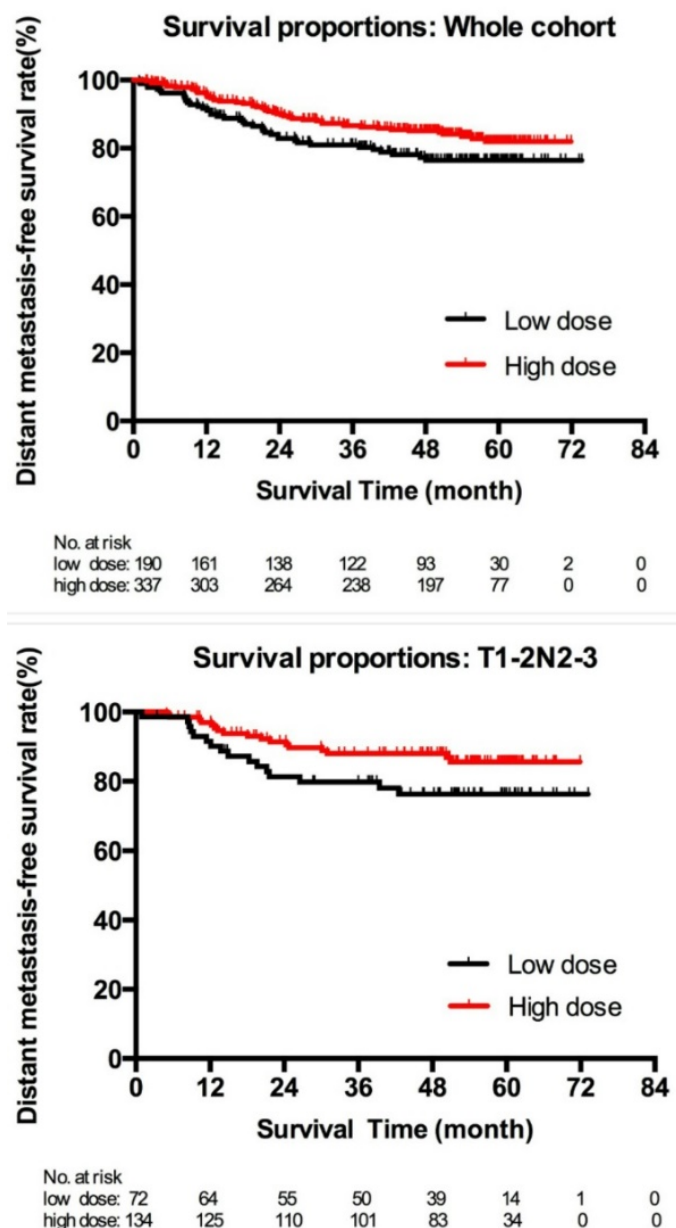

Table 6 Multivariate analyses of various clinical factors on survival rates among those treated with concurrent chemoradiation $(\mathrm{N}=278)$ and the subgroup treated with induction chemotherapy plus concurrent chemoradiation $(\mathrm{N}=244)$

\begin{tabular}{|c|c|c|c|}
\hline Survival & P value & HR & $95 \% \mathrm{CI}$ \\
\hline \multicolumn{4}{|l|}{ CCRT cohort } \\
\hline \multicolumn{4}{|l|}{ LRFS } \\
\hline Gender & 0.075 & 0.371 & $0.124-1.105$ \\
\hline RT Boost & 0.004 & 3.013 & $1.430-6.347$ \\
\hline \multicolumn{4}{|l|}{ RRFS } \\
\hline RT Boost & 0.001 & 5.266 & $2.032-13.652$ \\
\hline \multicolumn{4}{|l|}{ DMFS } \\
\hline Total DDP dose & 0.008 & 0.427 & $0.228-0.801$ \\
\hline \multicolumn{4}{|l|}{ OS } \\
\hline Age & 0.086 & 1.750 & $0.925-3.313$ \\
\hline \multicolumn{4}{|c|}{ The subgroup of induction $\mathrm{CT}+\mathrm{CCRT}$} \\
\hline \multicolumn{4}{|l|}{ RRFS } \\
\hline RT Boost & 0.007 & 4.821 & $1.530-15.193$ \\
\hline \multicolumn{4}{|l|}{ DMFS } \\
\hline Total DDP dose & 0.005 & 0.372 & $0.186-0.743$ \\
\hline \multicolumn{4}{|c|}{$\begin{array}{l}\text { Age }(>48 / \leq 48) \text {, gender ( } \mathrm{F} / \mathrm{M}) \text {, stage subgroup (classified parameter), treatment strategy, } \\
\text { cumulative dose of DDP }\left(300 \mathrm{mg} / \mathrm{m}^{2} /<300 \mathrm{mg} / \mathrm{m}^{2}\right) \text {, radiation boost (yes } / \text { no) were } \\
\text { included into multivariate analysis among all patients treated with concurrent } \\
\text { chemoradiation. }\end{array}$} \\
\hline \multicolumn{4}{|c|}{$\begin{array}{l}\text { Age }(>48 / \leq 48) \text {, gender }(\mathrm{F} / \mathrm{M}) \text {, stage subgroup (classified parameter), cumulative dose of } \\
\mathrm{DDP}\left(\geq 300 \mathrm{mg} / \mathrm{m}^{2} /<300 \mathrm{mg} / \mathrm{m}^{2}\right) \text {, radiation boost (yes/no) were included into multivariate } \\
\text { analysis in the subgroup treated with induction chemotherapy plus concurrent } \\
\text { chemoradiation. For LRFS and OS, no independent prognostic factor was found. }\end{array}$} \\
\hline $\begin{array}{l}\text { Abbreviation: } \mathrm{RT}=\mathrm{Ra} \\
\text { DDP=cisplatin. }\end{array}$ & otherapy; C & 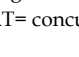 & nt chemoradiation; \\
\hline
\end{tabular}

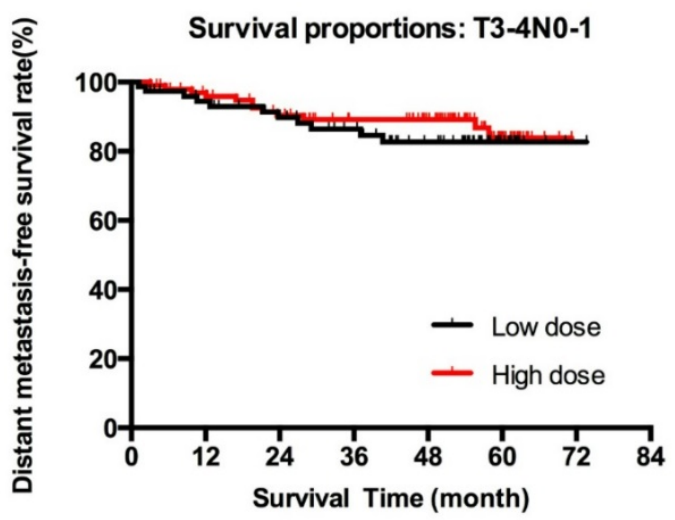
$\begin{array}{llllllll}\text { low dose: } \pi & 64 & 56 & 49 & 36 & 13 & 1 & 0 \\ \text { high dose: } 102 & 91 & 80 & 70 & 60 & 20 & 0 & 0\end{array}$

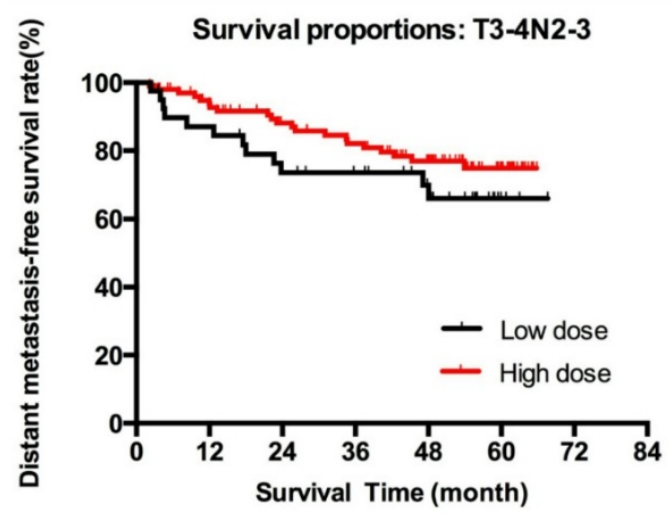

$\begin{array}{llllllll}\text { No. at risk } & & & & & & & \\ \text { low dose: } 41 & 33 & 27 & 24 & 18 & 3 & 0 & 0 \\ \text { high dose: } 101 & 87 & 74 & 67 & 54 & 23 & 0 & 0\end{array}$

Figure 1. Kaplan-Meier distant metastasis-free survivals (DMFS) by various stage subgroups and cisplatin dose subgroups. For the whole cohort of patients, significant benefit of DMFS was observed in the high-dose subgroup (figure $1 \mathrm{~A}, 82.0 \%$ vs. $76.5 \%, \mathrm{p}=0.029$ ). Of T1-2N2-3, high-dose subgroup was prone to have a better DMFS (85.7\% vs. $76.3 \%, p=0.069$, figure 1C). Of T3-4N0-1 and T3-4N2-3, no significant benefit of higher dose of cisplatin was observed (figure 1B and figure 1D). 
Survival proportions: Whole cohort
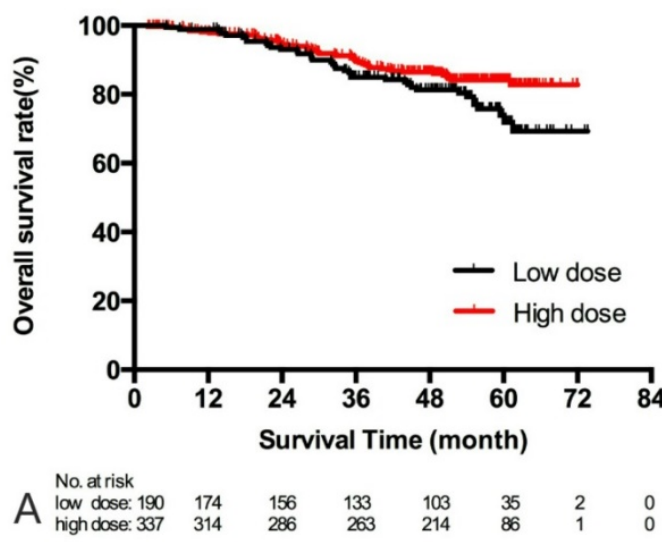

Survival proportions: T1-2N2-3

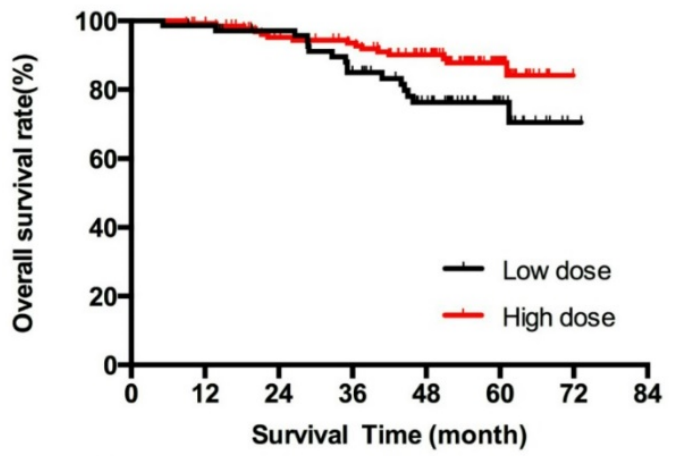

Cow dose: 72

$\begin{array}{lllllll}69 & 65 & 55 & 41 & 15 & 1 & 0\end{array}$

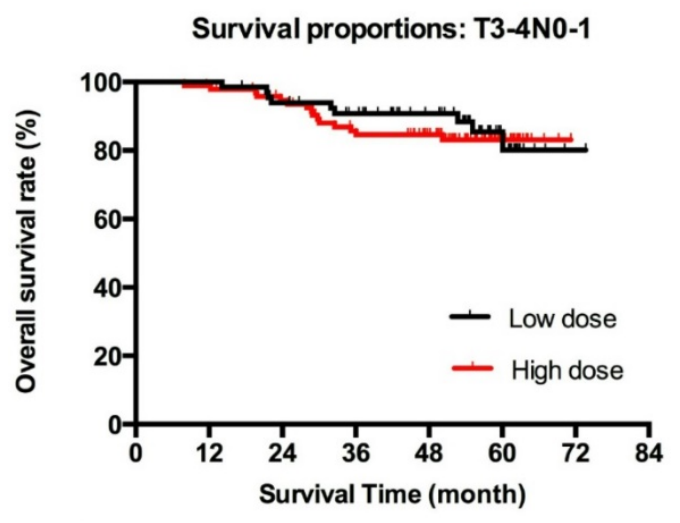

Survival proportions: T3-4N0-1

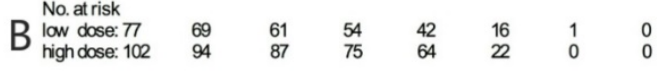

Survival proportions: T3-4N2-3

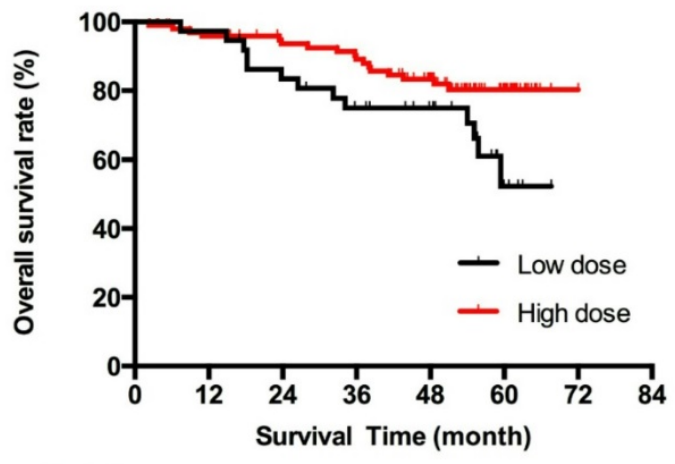

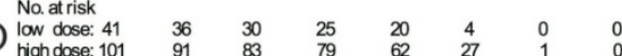

Figure 2. Kaplan-Meier overall survivals (OS) by various stage subgroups and cisplatin dose subgroups. For the whole cohort of patients, significant benefit of OS was observed in the high-dose subgroup (figure $2 \mathrm{~A}, 84.1 \%$ vs. $74.0 \%, \mathrm{p}=0.028$ ). Of $\mathrm{Tl}-2 \mathrm{~N} 2-3$, high-dose subgroup was associated with a better OS $(87.8 \%$ vs. $76.3 \%$, $\mathrm{p}=0.041$, figure $2 \mathrm{C}$ ). Of T3-4N2-3, high-dose subgroup was associated with a better OS as well ( $80.3 \%$ vs. $52.3 \%, \mathrm{p}=0.032$, figure $2 \mathrm{D})$. Of T3-4N0-1, no significant benefit of higher dose of cisplatin was observed (figure 2B).

\section{Discussion}

In times of 2DRT, higher cumulative dose of cisplatin was demonstrated to correlate with improved local control. In the combined analysis of NPC 9901 and NPC 9902 using 2DRT, the dose of cisplatin during the concurrent phase had significant impact on locoregional failure and overall survival [2]. However, the benefit of higher dose of cisplatin was controversial in the setting of IMRT. Wei et al. [10] reported that cumulative cisplatin $>200 \mathrm{mg} / \mathrm{m}^{2}$ during concurrent phase improved DMFS and progression-free survival in a cohort of patients with IMRT. Guo et al. [4] reported that the cumulative dose of cisplatin $>200 \mathrm{mg} / \mathrm{m}^{2}$ was associated with improved DMFS and OS among who received IMRT and CCRT. However, Peng et al. [5] reported no significant difference of LRFS, DMFS and OS between $<240 \mathrm{mg} / \mathrm{m}^{2}$ and $\geq 240 \mathrm{mg} / \mathrm{m}^{2}$ dose subgroups. Of note, the cutoff value of $240 \mathrm{mg} / \mathrm{m}^{2}$ was suboptimal with a low AUC of 0.506. The inappropriate dose threshold may hamper further analysis. In addition, the baseline distributions of $\mathrm{T}$ and overall stage were imbalanced between the two dose subgroups in this study. Higher proportions of T4 and stage IV disease were in the high-dose subgroup, which may compromise the potential improvement by high-dose cisplatin.

In our study, we used the median dose of cisplatin as the cutoff value, which was generally accepted in statistical analysis. The baseline characteristics were equally distributed, except a higher proportion of male patients and a marginal higher proportion $(p=0.053)$ of boost irradiation in the high-dose subgroup. Despite these adverse prognostic factors for DMFS, the higher dose subgroup had a significantly better DMFS and OS. In addition, the benefit of high dose of cisplatin were repeatedly proved in the entire cohort, T1-4N-2-3 subgroup, as well as those treated with CCRT. Taken together, our study validated the prognostic value of higher dose of cisplatin $\left(\geq 300 \mathrm{mg} / \mathrm{m}^{2}\right)$ on distant control and overall survival for locally advanced NPC in the setting of IMRT. 


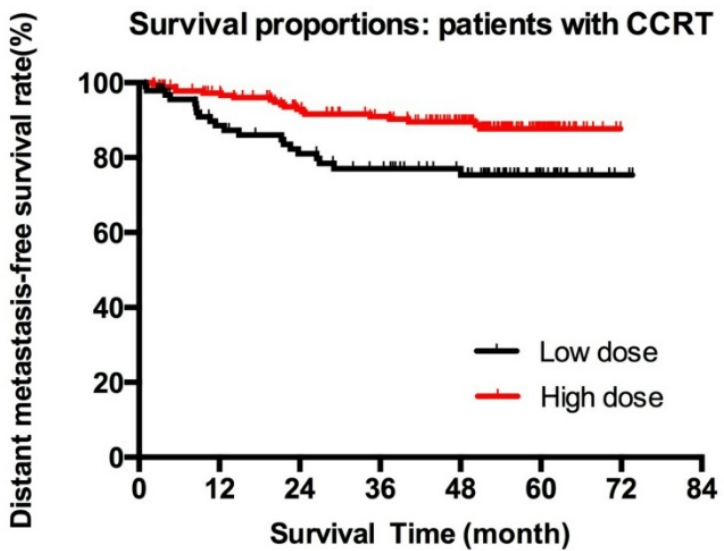

$\begin{array}{llllllll}\text { No. at risk } & & & & & & \\ \text { A low dose: } 94 & 74 & 64 & 56 & 45 & 19 & 2 & 0 \\ \text { high dose: } 184 & 166 & 146 & 134 & 108 & 44 & 0 & 0\end{array}$

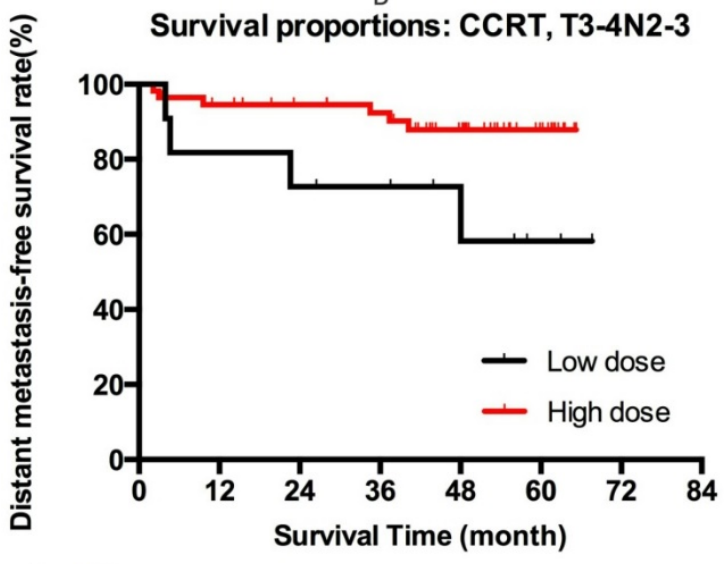

No. at risk

$\begin{array}{lrrrrrrr}\text { B low dose: } 13 & 9 & 8 & 7 & 5 & 2 & 0 & 0 \\ \text { high dose: } 57 & 49 & 45 & 42 & 32 & 14 & 0 & 0\end{array}$

Figure 3. Kaplan-Meier distant metastasis-free survivals (DMFS) of those treated with concurrent chemoradiation by various cisplatin dose subgroups. For the whole cohort treated with concurrent chemoradiation, significant benefit of DMFS was noted in the high-dose subgroup $(87.7 \%$ vs. $75.4 \%$, $P=0.004$, figure $3 A$ ). Similarly, high-dose subgroup tended to have a longer DMFS in patients with $\mathrm{T} 3-4 \mathrm{~N} 2-3$ disease treated with concurrent chemoradiation $(87.9 \%$ vs. $58.2 \%, p=0.034$, figure $3 B$ ).

In addition, the benefit of high-dose of cisplatin mainly displayed in reducing distant metastasis in times of IMRT, which was reported by previous researches $[4,10]$ as well as the present study. This was probably due to the shift of failure patterns after the application of IMRT. Since IMRT greatly improved local control, the major failure patterns for locally advanced NPC shifted to be distant metastasis [3]. Given the optimal local control [3, 11, 12], the gap of improvement may be narrowed. On the other hand, higher dose of chemotherapy plays a more prominent role in reducing distant metastasis.

Of note, locally advanced NPC is a heterogeneous entity. As was observed in our study, for T3-4N0-1, local relapse and distant metastasis accounted for half of the failures. Contrarily, in the subgroup of T3-4N2-3, the incidence of distant metastasis was three times of local recurrence. Given the higher risk of distant metastasis, the benefit of higher cumulative dose of cisplatin mainly derived from N2-3 subgroups. The reduction of distant metastasis was further transferred into improvement of overall survival in the entire cohort and the N2-3 subgroups. Cumulative dose of cisplatin $\geq 300 \mathrm{mg} / \mathrm{m}^{2}$ reduced $47.6 \%$ of risk of distant metastasis and $42.3 \%$ of deaths for the whole cohort of patients. As for the N2-3 subgroups, cumulative dose of cisplatin $\geq 300 \mathrm{mg} / \mathrm{m}^{2}$ reduced more than half the risk of distant metastasis and deaths. However, this benefit was not observed in T3-4N0-1 subgroups, either in univariate analysis or multivariate analysis. Based on this, we suggested the intensity of chemotherapy should be tailored for different subgroups of locally advanced NPC. For T1-4N2-3, adequate dose of cisplatin $\left(\geq 300 \mathrm{mg} / \mathrm{m}^{2}\right)$ during the entire chemoradiation should be emphasized. On the other hand, the dose threshold of $300 \mathrm{mg} / \mathrm{m}^{2}$ was not mandatory for T3-4N0-1 disease. De-intensification of treatment may be considered for T3-4N0-1 subgroups, probably in the way of lower dose of cisplatin during concurrent phase, or omission of concurrent chemotherapy in selected cases that achieved notable regression after induction chemotherapy. The rationale of de-intensification and optimal treatment strategy for T3-4N0-1 warrants further investigation.

Most of the previous studies enrolled patients treated with CCRT alone and evaluated the value of the cumulative dose of cisplatin during concurrent phase. However, more and more evidence supported the benefit of induction chemotherapy in locally advanced NPC [9, 13, 14]. Kong et al. [14] reported that TPF induction chemotherapy followed by CCRT provided promising outcome and acceptable toxicities in 2 phase II single-arm studies. Hui et al. [13] reported that TP induction chemotherapy followed by CCRT significantly improved progression-free survival in a phase II randomized trial, compared with CCRT alone. The updated meta-analysis of MAC-NPC group included 4806 patients from 19 trials. This analysis showed that induction chemotherapy brought significant benefit on progression-free survival $(7.7 \%$, 95\% CI: 1.3 to $14.1 \%)$ at 5 years, although the difference of OS did not reach a significant level. It should be noted that many studies in these analysis used a non-paclitaxel or non-docetaxel regimens, which may underestimate the absolute benefits of induction chemotherapy [15-17]. A multi-centered phase III randomized trial [9] has recently demonstrated that addition of TPF induction chemotherapy to CCRT significantly improved failure-free survival, DMFS and OS in locally advanced NPC. Taken together, induction chemotherapy was demonstrated to bring survival 
benefit to CCRT, mainly in reducing distant metastasis. Hence, when evaluating the influence of total dose of chemotherapy, it is reasonable to take the cumulative dose during the entire treatment into account, not only the part during concurrent phase. In addition, two clinical trials [18-20] proved the efficacy of induction chemotherapy plus adjuvant chemotherapy. A phase III randomized trial compared the efficacy of induction chemotherapy plus adjuvant chemotherapy with the efficacy of CCRT followed by adjuvant chemotherapy [18]. With a median follow-up of 60 months, the 5-year OS and DFS did not differ significantly between the two arms $[18,19]$. The 5-year OS of induction chemotherapy plus adjuvant for locally advanced NPC was $79.4 \%$. Of note, all patients in this study received 2DRT and attained an overall survival, which was comparable to the survival of locally advanced NPC treated with IMRT [3, 12]. Recently, a phase II single-armed clinical trial demonstrated promising result of those treated with GP induction chemotherapy plus GP adjuvant chemotherapy in locally advanced NPC [20]. The 5-year LRFS, DMFS and OS were $92.2 \%, 89.0 \%$ and $82.1 \%$, which was quite satisfactory for III-IVB patients.

To date, only one report from our institution has evaluated the influence of cumulative dose of cisplatin during the entire treatment and demonstrated that the cumulative dose $\geq 300 \mathrm{mg} / \mathrm{m}^{2}$ was associated with improvement of DMFS, DFS and OS. However, some scholars challenged that the lowest effectively cisplatin dose in our study may have been inflated by delivering cisplatin-based induction or adjuvant chemotherapy [5]. In addition, a part of patient in our study received induction followed by radiation or induction followed by adjuvant chemotherapy, which was not the standard strategy according to NCCN guidelines. In order to clarify this question, we focused on those received CCRT with or without induction/adjuvant chemotherapy and evaluated the impact of cumulative dose of cisplatin during entire treatment again. In consistence with the result from the whole cohort, more than $300 \mathrm{mg} / \mathrm{m}^{2}$ of cisplatin was a favorable prognostic factor for DMFS in those treated with CCRT, both in univariate and multivariate studies. Furthermore, more than $300 \mathrm{mg} / \mathrm{m}^{2}$ of cisplatin was also a favorable prognostic factor for DMFS in the subgroup treated with induction chemotherapy followed by CCRT.

One of our major limitations was the retrospective nature of the study. The baseline characteristics might be imbalanced and the treatment strategies might be not uniform. In our study, the baseline characteristics of the low-dose and high-dose subgroups were well balanced, except the difference of gender and the marginal difference of radiation boost. However, in the multivariate analysis adjusting various baseline parameters and treatment strategies, cumulative dose of cisplatin still was significant for OS and DMFS.

In conclusion, our study confirmed the prognostic value of cumulative dose of cisplatin during the entire treatment for locally advanced NPC and identified the benefit mainly derived from N2-3 patients. The benefit of higher cumulative dose maintained in those treated with CCRT. However, the subgroup of T3-4N0-1 did not benefit from higher cumulative dose, which suggested a need to tailor the intensity of chemotherapy based on various stage subgroups.

\section{Abbreviations}

NPC: nasopharyngeal carcinoma; CCRT: concurrent chemoradiotherapy; 2DRT: conventional two-dimensional radiotherapy; IMRT: intensity-modulated radiation therapy; GTV: gross tumor volume; CTV: clinical tumor volume; LRFS: local recurrence-free survival; RRFS: regional recurrence-free survival; DMFS: distant metastasis-free survival; OS: overall survival.

\section{Acknowledgements}

We thank our colleagues Dr. Zhou Xin, Dr. Shi Qi, Dr. Xing Xing, Dr. Yang Youqi and Dr. Jiang Wen for their help of collecting clinical data. This project is supported by the National Natural Science Foundation of China (Grant No. 81602372).

\section{Competing Interests}

The authors have declared that no competing interest exists.

\section{References}

[1] Loong HH, Ma BB, Leung SF et al. Prognostic significance of the total dose of cisplatin administered during concurrent chemoradiotherapy in patients with locoregionally advanced nasopharyngeal carcinoma. Radiotherapy and oncology : journal of the European Society for Therapeutic Radiology and Oncology 2012; 104:300-304.

[2] Lee AW, Tung SY, Ngan RK et al. Factors contributing to the efficacy of concurrent-adjuvant chemotherapy for locoregionally advanced nasopharyngeal carcinoma: combined analyses of NPC-9901 and NPC-9902 Trials. European journal of cancer (Oxford, England : 1990) 2011; 47:656-666.

[3] Lee AW, Ng WT, Chan LL et al. Evolution of treatment for nasopharyngeal cancer--success and setback in the intensity-modulated radiotherapy era. Radiotherapy and oncology : journal of the European Society for Therapeutic Radiology and Oncology 2014; 110:377-384.

[4] Guo SS, Tang LQ, Zhang L et al. The impact of the cumulative dose of cisplatin during concurrent chemoradiotherapy on the clinical outcomes of patients with advanced-stage nasopharyngeal carcinoma in an era of intensity-modulated radiotherapy. BMC cancer 2015; 15:977.

[5] Peng H, Chen L, Li WF et al. The Cumulative Cisplatin Dose Affects the Long-Term Survival Outcomes of Patients with Nasopharyngeal Carcinoma Receiving Concurrent Chemoradiotherapy. Scientific reports 2016; 6:24332.

[6] Ou X, Zhou X, Shi Q et al. Treatment outcomes and late toxicities of 869 patients with nasopharyngeal carcinoma treated with definitive intensity modulated radiation therapy: new insight into the value of total dose of cisplatin and radiation boost. Oncotarget 2015; 6:38381-38397. 
[7] Peng H, Chen L, Zhang Y et al. Prognostic Value of the Cumulative Cisplatin Dose During Concurrent Chemoradiotherapy in Locoregionally Advanced Nasopharyngeal Carcinoma: A Secondary Analysis of a Prospective Phase III Clinical Trial. The oncologist 2016; [Epub ahead of print].

[8] Blanchard P, Lee A, Marguet S et al. Chemotherapy and radiotherapy in nasopharyngeal carcinoma: an update of the MAC-NPC meta-analysis. The Lancet. Oncology 2015; 16:645-655.

[9] Sun Y, Li WF, Chen NY et al. Induction chemotherapy plus concurrent chemoradiotherapy versus concurrent chemoradiotherapy alone in locoregionally advanced nasopharyngeal carcinoma: a phase 3, multicentre, randomised controlled trial. The Lancet. Oncology 2016; 17:1509-1520.

[10] Wei W, Huang Z, Li S et al. Pretreatment Epstein-Barr virus DNA load and cumulative cisplatin dose intensity affect long-term outcome of nasopharyngeal carcinoma treated with concurrent chemotherapy: experience of an institute in an endemic area. Oncology research and treatment 2014; 37:88-95.

[11] Lin S, Pan J, Han L et al. Update report of nasopharyngeal carcinoma treated with reduced-volume intensity-modulated radiation therapy and hypothesis of the optimal margin. Radiotherapy and oncology : journal of the European Society for Therapeutic Radiology and Oncology 2014; 110:385-389.

[12] Sun X, Su S, Chen C et al. Long-term outcomes of intensity-modulated radiotherapy for 868 patients with nasopharyngeal carcinoma: an analysis of survival and treatment toxicities. Radiotherapy and oncology : journal of the European Society for Therapeutic Radiology and Oncology 2014; 110:398-403.

[13] Hui EP, Ma BB, Leung SF et al. Randomized phase II trial of concurrent cisplatin-radiotherapy with or without neoadjuvant docetaxel and cisplatin in advanced nasopharyngeal carcinoma. Journal of clinical oncology : official journal of the American Society of Clinical Oncology 2009; 27:242-249.

[14] Kong L, Hu C, Niu X et al. Neoadjuvant chemotherapy followed by concurrent chemoradiation for locoregionally advanced nasopharyngeal carcinoma: interim results from 2 prospective phase 2 clinical trials. Cancer 2013; 119:4111-4118

[15] Chan AT, Teo PM, Leung TW et al. A prospective randomized study of chemotherapy adjunctive to definitive radiotherapy in advanced nasopharyngeal carcinoma. International journal of radiation oncology, biology, physics 1995; 33:569-577.

[16] International Nasopharynx Cancer Study Group; VUMCA I Trial. Preliminary results of a randomized trial comparing neoadjuvant chemotherapy (cisplatin, epirubicin, bleomycin) plus radiotherapy vs. radiotherapy alone in stage IV(> or $=\mathrm{N} 2, \mathrm{M} 0$ ) undifferentiated nasopharyngeal carcinoma: a positive effect on progression-free survival. International journal of radiation oncology, biology, physics 1996; 35:463-469.

[17] Chua DT, Sham JS, Choy D et al. Preliminary report of the Asian-Oceanian Clinical Oncology Association randomized trial comparing cisplatin and epirubicin followed by radiotherapy versus radiotherapy alone in the treatment of patients with locoregionally advanced nasopharyngeal carcinoma. Asian-Oceanian Clinical Oncology Association Nasopharynx Cancer Study Group. Cancer 1998; 83:2270-2283.

[18] Xu T, Hu C, Zhu Get al. Preliminary results of a phase III randomized study comparing chemotherapy neoadjuvantly or concurrently with radiotherapy for locoregionally advanced nasopharyngeal carcinoma. Medical oncology (Northwood, London, England) 2012; 29:272-278.

[19] Xu T, Zhu G, He X et al. A phase III randomized study comparing neoadjuvant chemotherapy with concurrent chemotherapy combined with radiotherapy for locoregionally advanced nasopharyngeal carcinoma: updated long-term survival outcomes. Oral oncology 2014; 50:71-76.

[20] Wu M, He X, Hu C. Long-Term Results of a Phase 2 Study of Gemcitabine and Cisplatin Chemotherapy Combined With Intensity Modulated Radiation Therapy in Locoregionally Advanced Nasopharyngeal Carcinoma. International Journal of Radiation Oncology - Biology - Physics 2016; 96:S85-86. 